\title{
Corrections to: Quantitative Geosciences: Data Analytics, Geostatistics, Reservoir Characterization and Modeling
}

\section{Corrections to: Y. Z. Ma, Quantitative Geosciences: Data Analytics, Geostatistics, Reservoir Characterization and Modeling, https://doi.org/10.1007/978-3-030-17860-4}

The original version of the book was inadvertently published with certain errors. These have now been corrected.

1. On page 41: The prospect's acerage was inadvertently mentioned as $50 \times 50$ $\mathrm{km}^{2}$. This has now been corrected as below and the sentence now read as:

"a prospect's acreage is areally $50 \times 70 \mathrm{~km}^{2}$ "

2. On page 226: The second figure caption (Fig 9.15: (b) Histogram of bulk volume of oil (product of $S_{o}$ and Phie or SoPhie)) in Chapter 9 has been updated as "SoPhie"

3. On page 308: Equation 13.9 has been corrected as below

$\gamma(h)=c\left[1-\exp \left(\frac{-3 h^{2}}{a^{2}}\right)\right]$

4. In page 384: Equation 16.22 in Chapter 16 has been inadvertently mentioned as $s_{m}{ }^{2}=\operatorname{Variance}\left(m^{*}\right)=-\mu_{m}=\left(\boldsymbol{u}^{t} \boldsymbol{C}_{z z}{ }^{-1} \boldsymbol{u}\right)^{-\mathbf{1}}$. This has now been corrected as below in the book.

$e_{m}{ }^{2}=\operatorname{Variance}\left(m^{*}\right)=-\mu_{m}=\left(\boldsymbol{u}^{t} \boldsymbol{C}_{z z}{ }^{-1} \boldsymbol{u}\right)^{-\mathbf{1}}$.

5. On page 386: The subscripts and superscripts are kept as they are in the equation 16.29 in Chapter 16.

6. On page 393: Equation 16.45 has been corrected as below:

${ }_{\text {es }} \sigma_{y_{i}}^{2}=\sigma_{y_{i}}^{2}-\left(c_{y_{i}}^{t} \boldsymbol{A}_{y_{i}}+L_{y_{i}}\right)$

The updated version of this book can be found at https://doi.org/10.1007/978-3-030-17860-4_2 https://doi.org/10.1007/978-3-030-17860-4_9 https://doi.org/10.1007/978-3-030-17860-4_13 https://doi.org/10.1007/978-3-030-17860-4_16 https://doi.org/10.1007/978-3-030-17860-4_17 https://doi.org/10.1007/978-3-030-17860-4 
7. On page 406: Equation 17.1 in Chapter 17 has been corrected as below.

Variance $\{P(x)\}=$ Variance $\left\{P^{*}(x)\right\}+\sigma_{s k}^{2}$

8. On page 414: The equation number has been inadvertently numbered as 17.11 . This has now been corrected as 17.13. 